\title{
Hombres y masculinidad ¿Un cambio de modelo? ${ }^{1}$
}

\author{
José María Valcuende del Río ${ }^{1}$, Juan Blanco López ${ }^{2}$ \\ ${ }^{1}$ Departamento de Antropología Social, Psicología Básica y Salud Pública, Universidad Pablo de \\ Olavide y Aula de Derechos Humanos y Facultad de Filosofía de la Universidad de Cuenca \\ (Proyecto Prometeo/SENESCYT). \\ 2 Departamento de Trabajo Social, Universidad Pablo de Olavide, Ctra. de Utrera, km. 1 41013, \\ Sevilla - 954349 200, España.
}

Autor para correspondencia: jose.valcuende@ucuenca.edu.ec

Fecha de recepción: 6 de noviembre de 2014 - Fecha de aceptación: 30 de enero de 2015

\begin{abstract}
Machismo é um sentimento que gosto definir como o de mandar nas emoções da pessoa que se penetra, seja física, seja idealmente. Com o corpo ou com as ideias. Sentimento de dominação do espaço social e dos afazeres. Comando sobre a lei costumeira e a lei positiva. Sentimento necessário, como o etnocentrismo, de pensar que somos os melhores, os que mais sabemos, os que entendemos o contexto e o definimos (Raul Iturra, 2006).
\end{abstract}

\section{RESUMEN}

En las últimas décadas hemos asistido a un importante impulso en las investigaciones centradas en el análisis de las masculinidades. Este hecho no es casual y se debe fundamentalmente a dos razones. Una de carácter político, relacionada con el cuestionamiento de una sociedad representada exclusivamente en términos masculinos. Una segunda razón de carácter científico, ¿si la feminidad debía ser explicada por qué no la masculinidad? Ambas razones han incidido en la desnaturalización de una supuesta masculinidad de carácter universal y en la profusión de investigaciones tendentes a desvelar: las formas sociales de construir a los hombres, los mecanismos de reproducción del poder inscritos en los cuerpos, los desiguales modelos sociales en torno a lo masculino, las relaciones de dominación que se producen entre hombres y mujeres, y entre los propios hombres. Este cuestionamiento teórico de "lo masculino" ha estado muy vinculado al papel de los movimientos sociales y especialmente del feminismo y los movimientos de liberación sexual, que han jugado un papel central en la redefinición del papel de hombres y mujeres, y por tanto en la búsqueda de nuevas alternativas a los modelos tradicionales, también entre los propios hombres.

En las páginas siguientes nos aproximaremos precisamente a la noción de masculinidad y masculinidades, incidiendo en su carácter relacional y cambiante, así como en sus distintas significaciones.

Palabras clave: Hombres, masculinidad, género.

\footnotetext{
1 Desde que los autores coordinaran el libro: Hombres. La construcción cultural de las masculinidades. Ed. Talasa. Madrid, 2003, han pasado 13 años, en los que se han desarrollado de forma importante líneas de trabajo e investigación en el ámbito de la masculinidad. En este texto se presenta, teniendo como base la reflexión que realizamos en esa obra, una revisión de esta temática, corrigiendo, revisando y ampliando algunos de los debates, especialmente en lo referido a la aparición de nuevos modelos y cuestionamientos sobre la visión tradicional de la masculinidad. Este trabajo que presentamos se ha realizado en el marco del Proyecto Prometeo (SENESCYT) y se inscribe en las líneas de investigación del LIESS (Laboratorio Iberoamericano para el Estudio Socio-histórico de las Sexualidades (Proyecto I+D FEM2011-27295). Ministerio de Economía y Competitividad del Gobierno de España). Dicho proyecto articula a investigadoras e investigadores de Argentina, Cuba, Brasil, Costa Rica, Ecuador, España, México y Perú.
} 


\begin{abstract}
In the last decades we have witnessed an important boost to research focusing on masculinities analysis. Far from being coincidental, this is due to two different reasons: A political one, related to the questioning of a society depicted exclusively in masculine terms; and a scientific other, related to the fact that, with femininity being questioned, Why should we not question masculinity? Both reasons have influenced the de-naturalization of a supposed universal masculinity and the proliferation of studies tending to reveal issues as: social ways of building men; power reproduction mechanisms engraved in bodies; unequal social models around the masculine issue, domination relationships between men and women, and between men themselves. This theoretical wondering around "the masculine" has been closely linked to social movements, specially feminisms and sexual liberation movements, which have played a central part in redefining women's and men's roles, and hence, in the quest of new alternatives to traditional models, also for men themselves.

In the following pages we actually approach the notions of masculinity and masculinities, emphasizing their relational and changing character, as well as their different meanings.
\end{abstract}

Keywords: Men, masculinity, gender.

\title{
1. LA NOCION DE MASCULINIDAD
}

Si hablamos de la masculinidad como modelo de dominación ${ }^{2}$ nuestra primera tarea es definir qué entendemos por una noción tan amplia como ambigua, para ello utilizaremos como punto de partida la propuesta por Kimmel (1997):

La masculinidad es un conjunto de significados siempre cambiantes, que construimos a través de nuestras relaciones con nosotros mismos, con los otros, y con nuestro mundo. La virilidad no es estática ni atemporal; es histórica; no es la manifestación de una esencia interior; es construida socialmente; no nos sube a la conciencia desde nuestros componentes biológicos; es creada en la cultura. La virilidad significa cosas diferentes en diferentes épocas para diferentes personas. Hemos llegado a conocer lo que significa ser un hombre en nuestra cultura al ubicar nuestras definiciones en oposición a un conjunto de otros, minorías raciales, minorías sexuales, y, sobre todo las mujeres (Kimmel, 1997: 49).

Aquí nos encontramos ya varias ideas importantes que desarrollaremos a lo largo de las páginas siguientes. La primera idea es que se nace con unas marcas corporales, que son reinterpretadas culturalmente en función de un modelo dicotómico y jerárquico de "masculinidad" y "feminidad, que reelabora/construye la genitalidad en clave cultural. La segunda idea es que la significación de lo que nosotros entendemos por masculinidad es cambiante en función de la temporalidad histórica y del contexto cultural. La tercera idea es que la noción de masculinidad se construye en nuestra cultura en oposición a grupos que han sido minorizados, y que por tanto no tienen la misma consideración social, básicamente: mujeres, niños, viejos, homosexuales..., grupos que de una u otra forma son considerados como no masculinos en algunos casos y en otros como menos masculinos. Es decir, la masculinidad es fundamentalmente una marca de poder y por tanto de dominación (Bourdieu, 2000). En todo caso es un concepto polisémico, que define estatus, territorios, procesos a los que hay que llegar, normas que nos indican las formas culturalmente aceptables de las interacciones sociales y las fronteras que no pueden traspasarse.

\footnotetext{
2 Para un análisis de las teorías en torno a la masculinidad ver Guasch (2012). En el caso latinoamericano encontramos aproximaciones interesantes en Hernández (2007). En 2010 este mismo autor coordina un monográfico sobre masculinidades en la Revista Sociotam, Vol XX.1. Todos estos textos son accesibles en internet.
} 
Los planteamientos críticos a estos presupuestos constructivistas señalarían que esto es la negación de la naturaleza como factor fundamental a la hora de entender las diferencias entre hombres y mujeres. Y efectivamente, en parte es así, y lo es porque lo que se hace es precisamente cuestionar ciertas visiones también culturales, como no podría ser de otro modo, sobre una supuesta idea de naturaleza, que encubre toda una serie de presupuestos ideológicos encaminados a legitimar más la desigualdad que la diferencia, pero peor aun, que pretenden evitar cualquier transformación que no esté dictada por esa nueva deidad, a partir de la cual se puede legitimar cualquier cosa. Al fin y al cabo, ¿qué es lo que no está en la naturaleza?

Hace un tiempo llegó a nuestras manos una documentación sobre un taller de género, pensado para jóvenes. En dicha documentación había una actividad que nos llamó la atención. Se proponía a los participantes del taller cerrar los ojos y pensar cuándo fue la primera vez que tuvieron conciencia de ser una mujer o de ser un hombre. Independientemente del interés que tiene esta práctica en relación a la forma en que nos reinventamos, y que recreamos nuestro pasado a partir de nuestro presente. Por mucho que cerremos los ojos será difícil conseguir recordar el momento primigenio en el que vimos la luz, en el que descubrimos con tristeza o alegría que éramos hombres, y es que probablemente ese momento no existe. Hay determinadas cosas que aparentemente no necesitan explicación, es casi cómo preguntarse cuándo tuvimos conciencia de que éramos un ser vivo. Y es que el sexo, como construcción cultural, se incorpora al individuo antes del nacimiento a partir del momento en el que una ecografía desvela si el nuevo ser humano tendrá un pene o una vagina, un hecho nada inocuo y que marcará de por vida su futuro, y de paso el propio embarazo de la madre ${ }^{3}$.

Tal vez si la pregunta que se realizaba en la práctica del taller hubiese sido cuál es tu primer recuerdo en el que te diste cuenta de que no eras un hombre o una mujer, entonces casi todos y todas recordaríamos instantes en el que nuestras formas de comportarnos, nuestras formas de sentir y de vivir no se ajustaban a un modelo homogéneo de masculinidad y de feminidad inscrito en el cuerpo; casi todos y todas recordaríamos momentos de contradicciones entre los modelos sociales impuestos e interiorizados desde la infancia y nuestra individualidad constreñida a toda una serie de normas explícitas e implícitas. Normas que hemos asumido para poder ser no tanto seres humanos, categoría ue en la interacción social no sirve de mucho, sino hombres o mujeres. Y es que sí que efectivamente, tal y como señala Kimmel (1997), la masculinidad se construye en la relación con los otros y con nosotros mismos, se redefine en función del contexto; también la masculinidad se recibe en un proceso que sin darnos cuenta nos introduce dentro de un orden social donde los cuerpos, los sentimientos, las acciones tienen un sexo definido socialmente. El antropólogo Pedro Cantero en un texto de una gran belleza que se titula "Hombrear" nos narra su propia experiencia en este proceso de aprendizaje:
Al abrigo de las enormes puertas carreteras del pajar de mis abuelos, solía refugiarme con alguna vecinilla para hacer muñecas, eran figuras de barro o estructuras de palo, vestidas con trapillos y pelos de cáñamo, si mi abuela no le daba mayor importancia, mozos y hombres lo veían con desagrado: "Mariquita...Eso es cosa de niñas", decían, y tal fue la presión que, sin saber cómo, deje de dar vida a aquellas figuras.

Ya mayor, profesor en un instituto de formación para educadores, quise que hombres y mujeres aprendieran a hacer muñecas de trapo. Al principio los chicos, sorprendidos, no sabían cómo acomodarse, qué hacer con la tela, cómo tramar los pespuntes holgadamente, afinar las puntadas sin demasiada torpeza, manejar aguja y dedal; sin embargo, superada esa etapa, daban forma a seres con los que jugar sin reproches. Empresa inútil me diréis, puede que sí, pero, al menos, un grupo de hombretones superaron ese margen sin que se les cayeran los huevos. De eso doy fe (Cantero, 2003: 53).

\footnotetext{
3 No es casual la preocupación que se manifiesta en muchas culturas por el sexo del futuro ser humano, que se ha traducido en una curiosa y larga lista de técnicas populares para adivinar el sexo antes del nacimiento. Igualmente significativas son las creencias que diferencian el cuerpo de la mujer si está embarazada de un niño o una niña, e incluso los inconvenientes y ventajas del propio embarazo en función del sexo. Pero no es menos interesante el análisis de la acción social cuando las formas de los cuerpos de los seres humanos no se corresponden a los modelos establecidos, tal y como señala Benavente (2013) en el caso de los intersexuales.
} 
El texto de Cantero es indicativo de toda una serie de transformaciones que se han ido produciendo en un modelo cambiante tanto desde el punto de vista histórico como desde el punto de vista cultural, pero antes de adentrarnos en dichos cambios conviene que nos acerquemos a la visión tradicional sobre la masculinidad. Una visión que sigue en buena medida vigente, tal y como señala Guasch:

\begin{abstract}
El individualismo insolidario postmoderno disuelve la noción de identidad, de cualquier identidad. Pero la masculinidad tradicional continúa existiendo porque en un momento de cambio social rápido ofrece a los varones una posibilidad de autoconceptuación y de socialización que continúa siendo importante, ya que a través de la relación con los similares se fortalece la autoestima mediante la pertenencia grupal. (Guasch, 2003: 122).
\end{abstract}

\title{
2. EL MODELO DOMINANTE DE MASCULINIDAD
}

Tradicionalmente hemos entendido la masculinidad como un conjunto de cualidades asociadas a un sexo. Unas cualidades "naturales" que deben ser recreadas y transformadas a través de una cultura que elabora un ser, que en contraposición a la mujer se nos presenta como complejo y diverso, es decir, como elaborado culturalmente. Y aquí radica una de las grandes diferencias entre nuestra forma de concebir la masculinidad y la feminidad. Si se considera que el hombre tiene que ser construido culturalmente, también se asume que la mujer lo es por naturaleza (no es preciso hacer nada más que tener la menstruación). De esta forma es la naturaleza la que explica por qué una mujer es femenina, mientras que es la cultura la que explica la elaboración social del hombre. Y este último en cuanto que es conformado por la cultura, tiene el "peligro" de dejar de ser... La masculinidad es un indicativo de poder, y por ello debe demostrarse constantemente. Y es que tener el cuerpo "adecuado" es necesario pero no suficiente, se requiere algo más...

El Diccionario de la Real Academia de la Lengua Española define la masculinidad como lo que es propio exclusivamente del varón. La masculinidad se entiende así como indisociablemente vinculada a la forma de los cuerpos. La noción de masculinidad, desde este punto de vista, podríamos explicarla de una forma sencilla pero especialmente gráfica: como todo lo que no tiene que ver con las mujeres. Es decir, es el continente el que acaba por definir el contenido ${ }^{4}$. Volvamos al diccionario para saber cómo se entiende no ya la masculinidad sino lo masculino: "Dícese del ser que está dotado de órganos para fecundar", suponemos que dichos órganos deben ser los testículos y el pene, aunque esto no está especificado... Lo masculino se vincula de una forma automática a la posibilidad de reproducción, partiendo desde una supuesta acción que tendrá sus efectos en un ser que no actúa sino que "espera" la fecundación. Un hombre desde niño tiene que aprender lo que significa "tener dos cojones", básicamente esto estaría recogido en la segunda acepción del diccionario: masculino significa "varonil, enérgico", mientras que el significado de "femenino" haría referencia al: "ser dotado de órganos para ser fecundado" o en otra breve definición, no por ello menos significativa:

\footnotetext{
4 Aquí nos encontraríamos con un debate realmente interesante. Por un lado, los planteamientos que defienden que la masculinidad no tiene unos contenidos precisos sino que se define fundamentalmente como una "oposición a". Por otro lado, las posturas que plantean que no es cierto que la masculinidad se defina sólo en negativo, sino que existen toda una serie de prácticas, creencias, etc. a partir de las cuales se la dota de plena significación. Probablemente ambas posturas no son incompatibles, ya que efectivamente lo masculino en función de los contextos se redefine y se le dota de significado, aunque también es verdad que las prácticas no sólo pueden ser definidas por ellas mismas, sino que adquieren plena significación en función de quienes las realizan. Esto se evidencia de una forma clara en el campo laboral donde determinadas actividades que se han "feminizado" han pasado a ser traducidas socialmente de forma muy diversa en función del sexo de los trabajadores y trabajadoras. Continente y contenido son indisociables, y aunque es preciso un contenido (elementos que dotan de significado a la masculinidad en función del contexto y grupo social) es la forma de los cuerpos (el continente) el que puede modificar su significación.
} 
"débil, endeble"5. No es extraño, por tanto, que en el significado de "feminidad", en su segunda acepción, se señale lo siguiente: "Estado anormal del varón en que aparecen uno o varios caracteres sexuales femeninos", debemos suponer que vinculados a la debilidad, la endeblez y otros rasgos de características similares que son, en definitiva, los que definen a las personas afeminadas: dícese del que en su persona, modo de hablar, adornos o acciones se parece a las mujeres.

La naturaleza predispone al hombre a la movilidad, a la acción, a fecundar... pero una cosa es la predisposición y otra la necesaria acción que debe corresponderse con "su naturaleza". Es precisamente en este segundo nivel donde el hombre debe demostrar que es un verdadero hombre: acción frente a pasividad, fuerza versus debilidad, firmeza contra pusilanimidad. La caracterización de la masculinidad y la feminidad adquieren significación en tanto listado de elementos contrapuestos y complementarios, en función de un modelo heterosocial dominante. Un modelo que entiende no de seres humanos autónomos sino de pareja, compuesta por dos "mitades" que llegarán a la plena realización cuando sus elementos jerárquicamente distintivos se complementen en la verdadera unión refrendada por la religión, el derecho y/o la costumbre: el matrimonio ${ }^{6}$.

Se es hombre en cuanto se es digno de sustentar un modelo cultural, legitimado desde una naturaleza que se nos muestra como inmutable; una naturaleza que dirige la vida y la interacción de los seres humanos, agrupados en función de las marcas de los cuerpos en hombres y mujeres, en seres nacidos para dominar y en seres nacidos para ser dominados, en seres que nacen para fecundar y en seres que nacen para ser fecundados... Al fin y al cabo, esta visión dominante de la masculinidad parte de las lecturas de los cuerpos, continúa en las relaciones sexuales, entendidas como no podría ser de otro modo, como relaciones de poder, y se amplía a todos y cada uno de los campos de lo social: el espacio, el trabajo, la familia, las relaciones sociales. Sabuco y Valcuende del Río (2003) analizan la vinculación entre las interpretaciones del cuerpo y el poder:

Parodiando a Gertrude Stein, el mensaje es que: una polla es una polla es una polla es una polla. Y si siempre ha sido así, así debe seguir siendo, no puede ser de otra manera. Ellos son lo que son porque tienen pene. La representación de un hombre es la de un ser pollado. Es el pene, en abstracto, el que de forma metonímica, se transforma en un símbolo de poder y prestigio social para reproducir la posición dominante de los hombres. En contraste, en las mujeres sus ovarios, hormonas, menstruaciones, embarazos, etc., apuntalan toda una serie de limitaciones que legitiman mecánicamente las representaciones dominantes sobre "la feminidad" (emoción, fragilidad, histeria, variabilidad, etc.); la extirpación de algunos órganos internos se conoce popularmente como "vaciarlas (Sabuco \& Valcuende del Río, 2003: 146).

Todos y cada uno de los ámbitos tienen sexo en función de la complementariedad a través de la cual legitimamos las desigualdades. Sin duda esto tiene mucho que ver con el mito de la heterosexualidad, tal y como la entiende Guasch ${ }^{7}$ : un modelo social de dominación a partir del que se estructura la interacción social.

Lejos de la visión de la masculinidad como una esencia atada a una supuesta naturaleza, muchos autores parten de su carácter histórico, cambiante, relativo y diverso. Sin duda, los planteamientos

5 Los procesos educativos son centrales en la conformación de los modelos de masculinidad y feminidad, como lo son los rituales ordinarios y extraordinarios a partir de los que se representan y por tanto se construyen socialmente los hombres y las mujeres. En Valcuende del Río (1999) podemos encontrar un análisis en la significación de algunos de estos rituales en el caso español.

6 El debate que ha producido a nivel internacional en relación al matrimonio entre homosexuales es significativo de hasta qué punto la idea de complementariedad de funciones está profundamente arraigada. El matrimonio de los homosexuales, aunque sea civil, molesta profundamente entre los dirigentes de algunas de las principales religiones, precisamente porque pone en cuestión dicha complementariedad en función del sexo, pero también porque desvincula la relación automática entre sexualidad y procreación (Valcuende del Río, 2006). Tal y como señalamos es precisamente este modelo el que está en la base de algunas de las formas de violencia no sólo contra los disidentes sexuales, sino también contra las mujeres (Valcuende del Río, 2004).

7 Para comprender la visión de la heterosexualidad como un modelo de dominación (Guasch, 2000). 
feministas han contribuido de una forma fundamental a redefinir el modelo dominante sobre la masculinidad. El resignificar el sentido social de la feminidad afecta directamente a la propia noción de masculinidad. El cuestionarse la categoría mujer significa en último término desestabilizar la supuesta frontera fija e inmutable que sirve de contraste y de límite a partir del cual expresar la identidad de los varones. Pero determinados discursos feministas han contribuido también a deconstruir las categorías a través de las cuales se sustentaba el modelo dominante de masculinidad, desnaturalizando las supuestas esencias al desnaturalizar también la base sobre la que se sustentaban: el sexo. El sexo no determina el género; el sexo, los cuerpos, son también construcciones culturales cambiantes fuertemente condicionados por los modelos culturales de masculinidad y feminidad. Modelos, por otro lado, indisociables de otras variables a través de las cuales regulamos la interacción social: la edad, la pertenencia étnica, la clase social, la religión, etc.

La masculinidad está claro que es una construcción cultural como lo es la feminidad, algo que ya comienza a atisbarse en el discurso antropológico en los años treinta con autoras como Mead (1973), cuando discute la existencia de una personalidad femenina o masculina de carácter universal.

Descubrí a tres tribus dentro de un área de un centenar de millas. En una de ellas, tanto hombres como mujeres se comportaban como consideramos que deben hacerlo las mujeres: con reacciones paternales y amorosas; en la segunda, ambos actuaban como consideramos que deben hacerlo los hombres: educando con brutalidad; y en la tercera, los hombres se comportaban de acuerdo con el modelo estereotipado que tenemos de las mujeres: eran astutos, se rizaban el pelo e iban de compras, mientras las mujeres eran compañeras enérgicas, decididas y no usaban adornos." En el prólogo a esta edición señala: Todo este material nos permite afirmar que muchos, si no todos, los rasgos de la personalidad que hemos llamado masculinos o femeninos van tan poco ligados al sexo, como el vestido, los ademanes y la forma de peinarse que una sociedad, en una época determinada, asigna a cada sexo (Mead, 1973: 9-10).

No existe una personalidad masculina universal, como tampoco existen unos mismos contenidos que unifiquen el "ser hombre". Un ser o un no ser que debe ser analizado siempre en una coyuntura histórica y social determinada. En el saber del "sentido común" el nacer hombre o mujer debe determinar la masculinidad o la feminidad, eso por lo menos a nivel de discurso, sin embargo la práctica manifiesta otra realidad muy distinta. Si la "masculinidad" es una realidad "natural", ¿por qué en la mayor parte de las culturas se desarrollan toda una serie de mecanismos tendentes a impedir que los varones se alejen del modelo de masculinidad imperante?

La masculinidad dominante es un modelo ideal y por tanto inalcanzable. Un modelo que debe ser interiorizado a nivel individual y que debe ser preservado a nivel colectivo para impedir que los nacidos para mantener la familia, ocupar el poder político y social no se olviden que estas funciones están inscritas en sus genitales. El varón, en este sentido, debe superar constantemente toda una serie de pruebas donde se pone de manifiesto que la masculinidad debe reafirmarse constantemente y en cualquier contexto. Dicho de otra forma, su cuerpo indica ya cuál debe ser su posición, pero esta posición hay que mantenerla día a día, ya que siempre existe el riesgo de contaminación de "lo femenino", de lo que está más allá de la identidad masculina.

En ese "mundo de hombres" es necesario demostrar que se es capaz de ejercer el poder, que se está preparado. Los seres humanos que tienen pene, tienen la marca del poder; pero es preciso que esa "naturaleza" (potencialidad) se evidencie a nivel social, se haga efectiva.

El falocentrismo de la sexualidad dominante se sustenta en un órgano que se representa como no controlado, independiente, que escapa a la norma, salvo a la del deseo (...). Una de las bases sobre las que se forja y reproduce este principio, en nuestro sistema social, es la corporización del poder, aunque el pene no se nombre. Hay muchas frases al uso donde el poder se sexualiza para mostrar la sumisión ("que te den", "vete a tomar por culo", "estás jodido", "a mamarla”, "me la han metido bien metida...") o cuando se amplían las referencias orgánicas para denotar la falta en su ejercicio ("no tienes 
cojones"), aunque por ello no se niega la posibilidad de ejercer un poder consustancial al hombre, un poder que viene dado ("ser un calzonazos" es, al fin y al cabo, revestir el poder, ocultarlo (Sabuco \& Valcuende del Río, 2003: 145).

El llegar a ser hombre o mejor dicho el llegar a ser un verdadero hombre, constituye una auténtica carrera de obstáculos tanto a nivel interno como externo. Una carrera de frustraciones que no tendrá final, condenada al fracaso en tanto que siempre se puede ser más hombre... en tanto que normalmente nuestra vida termina en la vejez. La realización suprema de este discurso dominante sólo la pueden realizar algunos varones, durante una parte de su vida, ya que en la niñez la masculinidad es un proyecto por hacer y la vejez marca un periodo que se aleja de la masculinidad entendida como poder. Goffman (1963) señala cómo han entendido los norteamericanos el modelo ideal de masculinidad:

Un joven casado, blanco, urbano, heterosexual norteño, padre protestante de educación universitaria, empleado a tiempo completo, de buen aspecto, peso y altura, con un récord reciente en deportes. Cada varón estadounidense tiende a observar el mundo desde esta perspectiva... Todo hombre que falle en calificar en cualquiera de estas esferas, es probable que se vea así mismo... como indigno, incompleto, e inferior (citado en Kimmel, 1997: 50).

Y es que efectivamente tal y como señala Kimmel (1997) la definición hegemónica de la masculinidad es la de un hombre en el poder, un hombre con poder y un hombre de poder. El proyecto de la masculinidad analizado por este autor se nos presenta como un proyecto de carácter excluyente, en tanto que excluye a las mujeres y a una buena parte de los hombres, que no responden al modelo dominante (grupos minorizados), a los que no se les permite ejercer dicho poder, porque no responden fielmente a la interpretación social de una naturaleza, reinventada a nivel social.

Pero al igual que este proyecto excluye a hombres y mujeres también es cierto que el modelo de masculinidad hegemónica es reproducido tanto por hombres como mujeres. No podemos caer en los planteamientos maniqueos que tienden a presentar esencialmente a la mujer como víctima y al hombre como verdugo, entre otras cosas porque reconocer dichos planteamientos significa asumir nuevamente la posición de inferioridad de las mujeres en relación a los hombres; reconocer un papel pasivo de las mujeres a lo largo de la historia; asociar los modelos sociales a los sexos de una forma mecánica; olvidarse que estamos haciendo referencia a una forma de entender las relaciones entre seres humanos que comparten hombres y mujeres, y de la que difieren también hombres y mujeres. Significa también asumir la visión androcéntrica que ha negado la presencia activa de las mujeres a lo largo de la historia o que simplemente no ha querido ver a las mujeres con poder. Al considerar a éste como esencialmente masculino, cualquier mujer con poder es simplemente negada o "masculinizada" . Sin embargo y partiendo de este hecho debemos reconocer que en el modelo dominante de masculinidad, en el que se ha sustentado la heterosexualidad, la mujer ha ocupado normalmente un plano instrumental y secundario. Y es que si por un lado los hombres se piensan en relación a los hombres tal y como indicaba Marqués cuando contaba en una ocasión una divertida anécdota de un amigo suyo que presumía "de haberse acostado con mujeres de hombres muy importantes"; las mujeres no se piensan así mismas en relación a las mujeres, también lo hacen a partir de una imagen reinventada por los hombres: lo femenino es lo que gusta a los hombres, tal y como señala MacKinnon (1987).

En este enfoque, el poder masculino adopta la forma social de lo que los hombres, como género, desean sexualmente, lo que se centra en el poder mismo, según se define

\footnotetext{
8 La mejor forma de descalificar a una mujer con poder es precisamente señalar que actúa como un hombre o incluso diciendo que "es peor que un hombre". Lo que para unos está permitido e incluso es elogiable, forma parte de su naturaleza, para otras es una forma de descrédito. Las mujeres no sólo tienen más dificultades de atravesar lo que algunas autoras denominan "el techo de cristal”, sino que además requieren un plus de inteligencia, de bondad, etc. etc. Y es que bajo estos planteamientos en ocasiones lo que preocupa no es cómo las mujeres ejerzan el poder, sino que las mujeres estén en un lugar que no les corresponde, considerado esencialmente "masculino" y "masculinizador".
} 
socialmente. En los países capitalistas, esto incluye la riqueza. La masculinidad consiste en tenerla, la feminidad en no tenerla. [La masculinidad antecede a lo masculino al igual que la feminidad antecede a lo femenino y el deseo sexual masculino define a ambos]. Especificamente, "mujer" está definida por lo que el deseo masculino requiere para su excitación y satisfacción y ésta es socialmente tautológica con "sexualidad femenina" y "el sexo femenino". En las formas de trato permitidas hacia una mujer, en las formas socialmente no consideradas violaciones sino adecuadas para su naturaleza, se encuentran los detalles de los intereses y requisitos sexuales masculinos. [En el paradigma sexual concomitante, las normas imperantes de atracción expresión sexual están fusionadas con la formación y la afirmación de la identidad de género de modo que la sexualidad equivale a la heterosexualidad y equivale a la sexualidad del dominio (masculino) y la sumisión (femenina)] (MacKinnon, 1987).

En http://www.programamujerescdh.cl/media/publicaciones/pdf/18/53.pdf. Revisado el 16 de octubre de 2013.

Lo importante en la carrera de obstáculos que representa el proyecto de la masculinidad hegemónica es tener la aprobación de otros hombres, la mujer es solamente un instrumento. No es extraño que incluso la propia conceptualización de los distintos ámbitos del ejercicio del poder como: privados y públicos, productivos y no productivos, etc., responda precisamente a un modelo dominante que tiende a ocultar a los grupos minorizados, a aquellos que les corresponde ocupar otras esferas que no han sido ni deben ser visibles. No es casual que sean las mujeres las que inmediatamente han sido sumergidas en los ámbitos privados, como también lo han sido los y las homosexuales. Lo privado contribuye de forma fundamental a mantener el sistema ocultando acciones y hechos, prácticas que no se corresponden con los modelos, que existen pero no pueden ser públicas ${ }^{9}$. Así lo reconoce Llamas (1998):
El confinamiento del "sexo erróneo" (o del placer y el afecto "desviados" en la ignominia de la esfera privada supone su desvinculación radical con respecto a otros ámbitos de análisis de la realidad. Ese sexo es entonces psicología, pero no sociología, economía o política; es naturaleza o biología, pero no cultura o historia. El sexo disidente, como quintaesencia de lo privado, de lo individual, de lo presocial, se construye como "femenino" y como "homosexual"; y se asocia con el consumo y no con la producción. Correlativamente "lo masculino-heterosexual" se asocia con los valores públicos (Padgup, 1992). Las implicaciones que en todos los ámbitos de la vida tiene la sexualidad (los privados tanto como los públicos; los individuales tanto como los colectivos) vienen a demostrar que estas asociaciones son espurias. La condición del ejercicio de actividades públicas reconocidas como transcendentes reside en la adecuación inmaculada de esa vida menos privada (aunque tampoco manifiestamente sexual) al modelo social propiamente reconocido, culturalmente promocionado, económicamente "productivo", moralmente alabado, políticamente "conservador" (Llamas, 1998).

Efectivamente, el modelo social propiamente reconocido y culturalmente promocionado, se hace explícito en todos los ámbitos de la vida social y es reproducido a través de toda una serie de rituales de carácter ordinario y extraordinario. Muchos han sido los trabajos antropológicos a partir de los cuales se han estudiado los rituales de reproducción de la masculinidad, tanto en culturas "exóticas" como en culturas próximas. Los rituales tienen un carácter "conformador", suponen una forma de mostrar, producir, simbólicamente y de forma pública una transformación y de aceptar individualmente una nueva situación definida socialmente. En muchas culturas no basta con tener una

\footnotetext{
9 No es extraño tal y como señala Valcuende del Río (2010) que los homosexuales hayan debido conformar sus espacios y sistemas de redes al margen de los ámbitos laborales y familiares, como mecanismo de defensa, resistencia y socialización.
} 
edad para llegar a ser hombre, es la superación de una prueba, más o menos dolorosa, la que transforma a un ser humano en masculino:

El individuo entra en un orden (su propia sociedad), se sitúa en él y progresa hasta el final de su existencia. La iniciación masculina realiza la sociabilización, es el "verdadero" nacimiento, el acceso a un doble estado de realización en el medida en que el niño es considerado bajo la forma de un ser incompleto; consagra la madurez física, marca el cuerpo y confiere a la sexualidad su disciplina; implica revelaciones y enseñanzas, el ingreso en el conocimiento y la imposición de una moral sin lo cual es imposible valer socialmente. Por un simbolismo utilizado a menudo, la iniciación masculina se vive y manifiesta como una muerte (de la infancia y el mundo de las madres) y un nacimiento (de la madurez y el mundo social) simbólicos; es la mímica ritual del alumbramiento de los hombres hechos para adoptarse a la sociedad que los acoge (Balandier, 1998: 31-32).

Tal y como señala Cantero los niños deben aprender a vivir en un mundo de hombres que se convertirá en su mundo, en un proceso que se define a través de las rupturas (Cantero, 1996) ${ }^{10}$. El espacio del reconocimiento social exige una competencia constante, manifestar una posición y un estatus, que se traducen en nociones como la imagen pública, el honor, el pundonor.... en oposición a la virtud "femenina" de "la honra", tal y como señala Bourdieu en sus estudios sobre la Cabilia. El verdadero hombre debe mantener una imagen constantemente defensiva ante los demás para preservar su honor, su imagen, que también depende en buena medida de la acción de "sus" hijos y de "su" mujer, convertidos en monedas de transacción, que adquieren valor frente a los otros varones. El honor ha formado parte de eso que definimos como masculinidad, de esa necesidad constante de los varones de reafirmarse públicamente, ante las mujeres pero sobre todo ante los que dan sentido a la definición de masculinidad, tal y como señalamos anteriormente: los otros hombres.

El temor a que otros nos saquen ventaja se traduce en la creación de toda una serie de escudos tendentes a ocultar todo aquello que manifieste debilidad, que ponga en cuestión que estamos más acá de la frontera de la masculinidad. El aprendizaje de la hombría, es decir de la capacidad de gestionar el poder, de la posibilidad de dominar a otros seres humanos y, en último término, de tener la capacidad de ejercer la violencia implica un proceso de autocontrol de los aspectos que manifiesten vulnerabilidad. Kaufman (1995) afirma:

En términos más concretos, la adquisición de la masculinidad hegemónica (y la mayor parte de las subordinadas) es un proceso a través del cual los hombres llegan a suprimir toda una gama de emociones, necesidades y posibilidades, tales como el placer de cuidar a los otros, la receptividad, la empatía y la compasión, experimentadas como inconsistentes con el poder masculino. (...) Eliminamos estas emociones porque podrían restringir nuestra capacidad y deseo de autocontrol o de dominio sobre los seres humanos que nos rodean y de quienes dependemos en el amor y en la amistad. Las suprimimos porque llegan a estar asociadas con la feminidad que hemos rechazado en nuestra búsqueda de la masculinidad (Kaufman, 1995: 150) ${ }^{11}$.

Ciertamente en los modelos dominantes sobre la masculinidad existe un auténtico terror a la contaminación de lo femenino, porque realmente si no en el discurso sí en la práctica es muy fácil

10 Cantero analiza el proceso de construcción social de la masculinidad a través de la fiesta de quintos en una población de la sierra de Huelva (Andalucía/España). Los rituales de quintos estaban muy extendidos en la península y eran asociados a la fase previa de incorporación al servicio militar. Un periodo central que implicaba la asunción por parte del varón de las responsabilidades públicas y la mayoría de edad. Actualmente las celebraciones de quintos siguen subsistiendo en algunas poblaciones, pese a que el servicio militar ya no es obligatorio.

11 Se puede acceder a una revisión corregida y ampliada del texto citado en: http://www.michaelkaufman.com/wp-content/uploads/2008/12/los-hombres-el-feminismo-y-las-experiencescontradictorias-del-poder-entre-los-hombres.pdf. Consultado el 15 de octubre de 2013. 
alejarse de un modelo imposible y ficticio, no por ello menos real que marca lo que habíamos definido anteriormente como una meta inalcanzable. Un modelo vigilado en la familia, en la calle, en el trabajo... Ya en la adolescencia tal y como nos señala Kimmel (1997) "aprendemos que nuestros pares son un tipo de policía de género, constantemente amenazando con desenmascararnos como afeminados como poco hombres". El niño, mientras siga en el mundo femenino, no es hombre; el hombre que mantenga relaciones sexuales con otros hombres es menos hombre; el hombre que no manda en la casa pierde los pantalones; el hombre que deja de trabajar se aleja del modelo de masculinidad, como también se aleja el hombre que manifiesta sus sentimientos, el viejo que pierde la fuerza física... Alberto del Campo señala cómo, en un ámbito tan significativo como el fútbol, se establecen toda una serie de estrategias tendentes a desestabilizar al contrario, precisamente cuestionando su masculinidad, o lo que es equivalente su sexualidad (léase heterosexualidad). Un ejemplo que es sintomático de la rivalidad entre varones no sólo en los ámbitos supuestamente "deportivos":

Una de las maneras más recurrentes de desequilibrar a tu contrario en un partido de fútbol es insinuarle de una manera u otra su carácter afeminado u homosexual. Es una lección que cualquier aprendiz de Míchel ${ }^{12}$ nos puede facilitar, pues desde las categorías alevines se aprende éste y otros trucos para ganarle la batalla psicológica al rival.

Escandalosa fueron las imágenes televisivas donde la cámara ponía su atención sobre el jugador merengue tocando ostentoriamente las partes a su marcaje Valderrama.

Escandalosa para los aficionados pues el hecho aunque motivo de burlas y bromas en los entrenamientos no supuso una novedad entre los futbolistas. Se había destapado para la afición una de las estrategias de lucha tácitamente admitidas por los jugadores pero ocultas al espectador habitual. Los tocamientos de Míchel incomodan y ponen nervioso a su víctima pues las connotaciones homosexuales de su acción desajustan el modelo de comportamiento masculino en el terreno de juego. Si el fútbol es cosa de hombres -léase heterosexuales- todo lo que rompa con el comportamiento masculino aprendido del oponente le situará en un marco de incertidumbre, de tensión (Campo, 2003: 91).

El futbolista es una de las diversas imágenes del héroe moderno, del guerrero que tiene que representar todos los atributos más característicos de la masculinidad ${ }^{13}$. El fútbol es un contexto más donde, al mismo tiempo que se representan determinados papeles, se construyen y reproducen ciertas formas de ser masculinos. Este deporte es, sin duda, una de las mejores metáforas para comprender la masculinidad y sus límites, así como el proceso de aprendizaje para llegar a ser un verdadero hombre.

La búsqueda de un modelo ideal y por tanto imposible conlleva el uso de la violencia simbólica y física, lo que a su vez, tal y como señala Bourdieu (2000), implica efectos normalmente ignorados, no sólo en los colectivos dominados, también entre los dominantes. El "ser y actuar" como hombre, supone asumir riesgos tanto personales como sociales que en determinadas ocasiones pueden constituir la expulsión del sujeto de la comunidad ideal de los "hombres de verdad". Si los valores no son meramente acumulativos, los asociados a la masculinidad provocan en muchos varones la exclusión no solo del grupo dominante sino de la propia sociedad y cultura que los crea y recrea. No deja de ser paradójico tal y como señala Blanco (2012) que el seguir el modelo de éxito social (la masculinidad) conlleve habitualmente el fracaso personal, cuando no la exclusión por el ejercicio de la autosuficiencia cuando no la violencia o por el escaso desarrollo de la capacidad de elementos vinculados discursivamente con la feminidad, como el cuidado.

\footnotetext{
12 Fue jugador del Real Madrid.

13 Guasch (2003) realiza un análisis de los tipos ideales de masculinidad a partir de la imagen de los ancianos, los guerreros, los efebos y los afeminados.
} 


\section{3. ¿MASCULINIDAD O MASCULINIDADES?}

Cuando hablamos de masculinidad no podemos olvidar que estamos hablando de un modelo construido en una sociedad concreta, en un tiempo histórico determinado. No podemos olvidar tampoco, que junto a este modelo dominante existen también otras formas de entender la masculinidad, es más, que la práctica cotidiana no necesariamente tiene que coincidir automáticamente con dicho modelo. Así lo señala el antropólogo portugués Val de Almeida (2000) cuando afirma:
Me parece central el uso de la noción de masculinidad hegemónica o sea no "el papel"
masculino, pero sí una variedad particular de masculinidad que subordina otras
variedades. Si la fisura entre las categorías "hombre" y "mujer" es uno de los hechos
centrales del poder patriarcal y de su dinámica, en el caso de los hombres la división crucial es entre la masculinidad hegemónica y varias masculinidades subordinadas (Connell, 1987). De aquí se sigue que las masculinidades son construidas no sólo por las relaciones de poder sino también por su interrelación con la división del trabajo y con los patrones de ligazón emocional. Por eso empíricamente se verifica que la forma culturalmente exaltada de masculinidad sólo corresponde a las características de un pequeño número de hombres (Val de Almeida, 2000).

Sin embargo, el que el modelo responda a pocos hombres no significa falta de eficacia de dicho modelo, en tanto que los "dominados", ya sean otros varones ya sean mujeres, lo interiorizan como el ideal a seguir, asumen la posición de inferioridad. Nuevamente retomamos a Val de Almeida (2000) al hablar de la hegemonía como una forma de dominación. Así la hegemonía aplicada al campo del género:
Se trata de la capacidad de imponer una definición específica sobre otros tipos de masculinidad (...) El concepto permite una concepción más dinámica de la masculinidad, entendida así como una estructura de relaciones sociales, en que varias masculinidades no hegemónicas subsisten, aunque reprimidas y auto-reprimidas por ese consenso y sentido común hegemónico, sustentado por los significados simbólicos incorporados (Val de Almeida, 2000: 155).

Discursos y prácticas son dos facetas no necesariamente coincidentes, en ese sentido se hace especialmente necesario profundizar en el conocimiento de la cotidianeidad que constantemente se reinterpreta desde lo que este autor define como "hegemonía".

La práctica pone de manifiesto que los hombres se muestran de formas muy diversas en función de los contextos. Hacer hincapié en la diversidad de prácticas puede contribuir a desvelar la diversidad de los dominantes dominados. Pero, sobre todo, puede contribuir a pensar en otras formas de entender las masculinidades o quizás las no masculinidades. Nuevos modelos que vayan más allá de los cambios puramente formales, un proceso realmente complejo más cuando el término de masculinidad se afianza en oposición a los otros, siendo un mecanismo de poder presente tanto en la acción cotidiana como en las instituciones (comenzando por la familia). Pero ¿qué queda hoy del modelo hegemónico después de los cuestionamientos realizados por los movimientos feministas, los movimientos de liberación sexual y algunos grupos de hombres? ¿Se han creado nuevos modelos alternativos? ¿En qué medida los hombres evidencian la necesidad de transformar dicho modelo? ¿Podemos hablar de crisis de la masculinidad?

Si entendemos "crisis" como transformación ésta parece evidente desde los años setenta en que se inician los estudios de hombres, independientemente de que en buena medida haya sido forzada por la lucha de las mujeres. Sin embargo, y antes de entrar en los "nuevos" modelos, conviene que nos detengamos, aunque sea de forma impresionista, en la situación actual.

El cuestionamiento de los roles y las actitudes de los hombres ha provocado reacciones diversas. Desde las reacciones que en una etapa de desconcierto buscan precisamente la reafirmación de los viejos modelos, a las que repudian el modelo tradicional, pasando por la indiferencia de los grupos de 
hombres que perciben que este problema no es suyo. Si tuviésemos que agrupar las respuestas a la crisis del modelo podríamos hacerlo en función de los siguientes grupos:

- La de los hombres "rebotados". El cuestionamiento del poder masculino y de los roles tradicionales de hombres y mujeres se ha traducido en este primer caso en una reacción tendente precisamente a reforzar el modelo tradicional. Los hombres "rebotados" se sienten amenazados por el papel cada vez más protagónico de las mujeres, y es que el miedo a lo femenino y a la feminización se ha manifestado en diversas direcciones con argumentos dispares. Se considera que las nuevas normativas que se han ido creando a nivel internacional perjudican en sus derechos a los hombres. Este es el caso de las leyes de violencia de género o bien de las sentencias judiciales en relación a los divorcios y custodia de los niños, habitualmente favorables a las mujeres. No es casual que sea en algunas asociaciones de hombres separados en los que este tipo de discursos cale con especial fuerza.

Pero en ocasiones el discurso de estos hombres "rebotados" es mucho menos sutil. Desde algunos planteamientos claramente vinculados con sectores neoconservadores se maneja un discurso claramente misógino, que sitúa a las mujeres en un claro papel de inferioridad, apelando a la solidaridad masculina para que ambos sexos ocupen el lugar que les corresponde. En muchos casos estas posturas están vinculadas a discursos religiosos, pero no sólo. De hecho algunos de sus argumentos se sustentan en los ámbitos académicos e "intelectuales", que recuerdan desde una idea supuestamente objetiva, a partir de los dictados de la naturaleza, cuál es el papel de hombres y mujeres; también cuál debe ser el papel de esos "menos" hombres definidos como homosexuales, cada vez más visibles, lo que genera una cierta incomodidad a los guardianes de la ortodoxia sexual, y una desestabilización de las masculinidades normativas, cuya reafirmación pasa, tal y como nos recuerda Guasch (2003) por la homofobia.

Curiosamente el peso de estas visión tradicionales ha ido ganando peso en algunos países, desde planteamientos que tienden a negar la plena igualdad de derechos de las minorías sexuales (prohibiendo a los disidentes sexuales el reconocimiento legal de sus familias, cuando no las expresiones públicas de afecto) a partir de la idea de una sexualidad reproductiva y heteronormativa. A su vez, nos encontramos con toda una serie de mecanismos de control sobre los cuerpos de las mujeres con normativas que siguen restringiendo, por ejemplo, los derechos sobre unos cuerpos controlados por los aparatos del Estado.

- La de los hombres que repudian el modelo dominante. Estos grupos al igual que los primeros son conscientes de la crisis del modelo hegemónico. Pero si el grupo anterior ante esta crisis reivindica el reforzamiento del modelo tradicional, en este caso se cuestiona dicho modelo y se asume el mismo como un problema tanto para las mujeres como para los propios hombres. Estos grupos, pese a ser minoritarios, han jugado y juegan un papel importante en algunas luchas vinculadas con el movimiento feminista, aunque son conscientes de los distintos puntos de partida tanto de hombres como mujeres. Consideran que el modelo de masculinidad ha sido un modelo de dominación que ha obligado a los propios hombres a renunciar a toda una serie de aspectos fundamentales, y plantean la necesidad de ser hombres de otra forma, en un proceso, nada sencillo, de renuncia a ciertos privilegios y de aprendizaje de nuevas formas de relación tanto entre los hombres, como entre estos y las mujeres. No es extraño que una buena parte de estos planteamientos críticos con los modelos tradicionales aparezcan en hombres vinculados a movimientos de liberación sexual, aunque también encontramos otros grupos que juegan cada vez un papel más importante, como son los grupos de hombres por la igualdad o contra la violencia de género, con una marcada influencia feministas en algunos casos, en los que se produce un proceso de reivindicación social, pero también de búsqueda personal de nuevos caminos, que permitan relaciones de carácter más igualitario.

- La de los hombres desconcertados. La imagen del macho proveedor no es ya una imagen políticamente correcta, ¿pero cuál es la alternativa?, ¿qué esperan las mujeres de los hombres y qué esperan los hombres de sí mismos? Una buena parte de los hombres tienen problemas 
por cuanto que no saben qué valores deben asumir en tanto hombres. Entre otras cosas porque el modelo de masculinidad dominante no sólo ha sido asumido por los varones, muchas mujeres plantean también expectativas contradictorias en sus relaciones con los primeros en su interacción cotidiana. Antes se tenía muy claro que cuando un hombre y una mujer debían atravesar una puerta primero debían pasar "las señoras", hoy día si se cede el paso puedes ser acusado de "machista", si pasas primero puedes ser acusado de "ordinario". Este ejemplo podríamos extrapolarlo a otros ámbitos: quién debe llevar la iniciativa en las relaciones sexuales, qué lenguaje debe ser utilizado, cómo realizar el reparto de papeles asignado tradicionalmente a los sexos... y mantenerse en los límites entre el denostado papel del "machito" o el no menos denostado papel de "blandengue".

- La de los hombres indiferentes. Hace unos años cuando organizábamos unas jornadas sobre masculinidad, preguntamos a un alumno si asistiría a las jornadas, la respuesta, más o menos, fue la siguiente: ¿Para qué? Yo no tengo problemas con mi masculinidad. Independientemente de que en muchos casos este tipo de respuestas encubre otros problemas, lo cierto es que una buena parte de los varones no los viven como tal. No podemos olvidar que el cuestionamiento de la masculinidad no ha afectado por igual a todos los hombres y mujeres, y que en la actualidad perviven de forma simultánea diversos modelos. En ocasiones cuando se señala que los cambios tanto en los roles como en los modelos es algo absolutamente generalizado, se olvida que existen muchos grupos sociales donde este cuestionamiento tiene un carácter absolutamente minoritario, y en que el modelo tradicional nunca ha dejado de estar vigente.

Si excluimos a este último grupo podemos categorizar las diferentes respuestas sociales, que han ido apareciendo en el último tercio del siglo XX y primeros años del XXI, por parte de los hombres organizados en asociaciones de la siguiente manera (Blanco, 2013) ${ }^{14}$.

\begin{tabular}{|c|c|c|c|}
\hline \multicolumn{4}{|c|}{ Categorización de los grupos de hombres. } \\
\hline \multirow{2}{*}{$\begin{array}{l}\text { Propuesta de Guasch. } \\
\text { "tipologías" }\end{array}$} & \multicolumn{2}{|c|}{ Propuesta de síntesis. } & \multirow{2}{*}{$\begin{array}{l}\text { Propuesta de Kimmel } \\
\text { "motivación" }\end{array}$} \\
\hline & $\begin{array}{l}\text { Orientación } \\
\text { conservadora }\end{array}$ & $\begin{array}{l}\text { Orientación } \\
\text { igualitarista }\end{array}$ & \\
\hline $\begin{array}{l}\text { Perspectiva } \\
\text { conservadora }\end{array}$ & $\begin{array}{l}\text { Grupos de } \\
\text { supremacía }\end{array}$ & & De supremacía masculina \\
\hline $\begin{array}{l}\text { Perspectiva pro- } \\
\text { feminista }\end{array}$ & & $\begin{array}{l}\text { Grupos pro- } \\
\text { feministas }\end{array}$ & $\begin{array}{c}\text { Igualitarios profeministas o } \\
\text { antisexistas }\end{array}$ \\
\hline \multirow[t]{2}{*}{$\begin{array}{l}\text { Perspectiva de } \\
\text { reivindicación de } \\
\text { derechos. }\end{array}$} & $\begin{array}{l}\text { Reivindicación } \\
\text { de derechos } \\
\text { sobre los hijos }\end{array}$ & $\begin{array}{l}\text { Reivindicación de } \\
\text { cuidados } \\
\text { compartidos }\end{array}$ & $\begin{array}{c}\text { Reivindicación de derechos } \\
\text { civiles. }\end{array}$ \\
\hline & $\begin{array}{l}\text { Grupos mito- } \\
\text { poéticos }\end{array}$ & Autoconciencia & $\begin{array}{l}\text { Autoconciencia o } \\
\text { reivindicación masculina }\end{array}$ \\
\hline $\begin{array}{r}\text { Grupos de liberación se } \\
\text { Feminis }\end{array}$ & $\begin{array}{l}\text { ual co-participes } \\
\text { del cuestionami }\end{array}$ & $\begin{array}{l}\text { nto al Movimiento } \\
\text { to. }\end{array}$ & Grupos de liberación sexual \\
\hline
\end{tabular}

Sin embargo en unos momentos en los que se reivindican nuevos modelos de masculinidad, algunos autores se plantean si esto es realmente necesario. Así lo señala Marques (2003) con su finísimo sentido del humor:

Cuando intentaba, en unos grupos de discusión realizados con el amigo José Angel Lozoya, explorar qué entendían por identidad masculina varones que rondaban los

\footnotetext{
${ }^{14}$ En Blanco (2013) se profundiza en el desarrollo y evolución de lo que se ha venido en denominar el Movimiento de Hombres en el caso español.
} 
cincuenta años, ante su falta de respuesta, transgredí la norma que pide al tutor del grupo intervenir demasiado activamente y les dije:

"Esto era un sevillano que se sentía muy sevillano. Le gustaba vestirse de corto, pasear a caballo por el Real de la Feria, tomar finos, tocar la guitarra, cantar sevillanas y deleitar a sus contertulios con un humor fino y ágil. Un día enronqueció y tuvo que dejar de cantar. Se llevó un disgusto pero siguió considerándose muy sevillano. Otro día el médico le quitó el fino, pero el siguió paseando por la Feria y considerándose muy sevillano. Tiempo después cayó del caballo, pero él siguió sintiéndose igual. Y así tuvo que dejar de tocar ... ¿Cuándo creen ustedes que dejó de ser sevillano? Bueno. Esto es un cuento para que me digan ustedes ¿cuándo un hombre deja de ser un hombre, todo un hombre, qué tiene que hacer, o permitir...?" (Marqués, 2003: 204).

Esta reflexión puede servirnos para aproximarnos a los nuevos modelos sobre la masculinidad y a las transformaciones que se están produciendo en los últimos años. Está claro que ha habido cambios sustanciales en nuestra forma de entender las relaciones entre hombres y mujeres, por lo menos en los discursos. Hoy ya no es políticamente correcto el decir que uno es un machista o que considera inferiores a las mujeres o a los homosexuales. Tampoco lo es, decir que los hombres no trabajan en la casa o no cuidan a los niños. En este proceso de transformación los hombres, en líneas generales, no parecen encontrar nuevos modelos de referencia. La desorientación y contradicciones entre los modelos tradicionales y los nuevos modelos es así una constante. Los hombres no saben, en muchas ocasiones, cómo representarse ante los otros y ante sí mismos ${ }^{15}$. El problema no solo radica en esa falta de modelos alternativos. Se complejiza si analizamos cuales son las características, valores y prácticas sociales asociadas con el éxito social y profesional que no difieren sustancialmente del modelo de masculinidad hegemónica: el ejercicio del poder, la capacidad de proveer, la no dependencia y la competencia entre iguales. Valores, aptitudes y actitudes que se contradicen tanto con las criticas fundamentadas y demandadas de los pensamientos feministas y anti-sexistas, como con los discursos políticamente correctos imperantes en nuestra sociedad sobre la "nueva imagen" del hombre, o con las reivindicaciones de unas nuevas formas de ser y ejercer la masculinidad.

Pese a esta falta de nuevos modelos que sirvan de forma general, nos encontramos con algunos emergentes, que llegaron a tener una cierta proyección mediática, este es el caso de los denominados metrosexuales. Fijémonos en este aparente nuevo modelo de masculinidad, en el que se nos muestra a un hombre más dulce y sensible, preocupado por el cuidado de su cuerpo y por el mantenimiento de ciertas formas sociales. Este modelo apunta así a una cierta trasgresión de de las fronteras de la feminidad, o lo hace por lo menos de forma similar a cierto modelo de homosexualidad masculina. Al fin y al cabo la estética metrosexual coincide, en buena medida, con la reinvención social de los gays, a los que se les presupone también una especial sensibilidad, detallismo, la obsesión por el cuidado su cuerpo $^{16}$, etc. Entonces la pregunta es ¿por qué inventar un modelo que está inventando? Desde nuestro punto de vista, precisamente para no transgredir la frontera que parece más fija en el modelo de la masculinidad dominante: la homosexualidad (Guasch, 2000).

Un metrosexual podría ser un gay, si el metrosexual no se encargase de decir qué es y qué no es. Entre otras cosas porque un gay sólo puede ser eso, gay (en el mejor de los casos, maricón en el peor), pero un metrosexual no quiere ser confundido. En este sentido, la metrosexualidad reproduce la lógica de la masculinidad dominante al establecer las fronteras entre los verdaderos hombres y los menos hombres, en este caso, los homosexuales. Algo que observamos en la publicidad sobre productos cosméticos destinados a un público masculino en la que se destaca, más que la calidad del producto, la verdadera masculinidad de los hombres que a pesar de usar "cosas de mujeres" no dejan de ser hombres de verdad, reforzando actitudes hipermasculinas y comportamientos que serían inadmisibles socialmente en otras circunstancias, para evitar aparecer como feminizados.

15 Blanco (2003) analiza con más detenimiento las contradicciones que se generan entre comportamientos y expectativas de y sobre los varones.

16 Para una crítica a la denominada cultura gay ver Sabuco \& Valcuende del Río (2003). 
Pero no es sólo ante los homosexuales donde se crean nuevas fronteras. En páginas anteriores señalábamos que la definición de masculinidad es la de un hombre con poder, un hombre de poder, un hombre en el poder. No es casual que el modelo de metrosexual se construya a partir de los nuevos héroes, los "gladiadores" de la modernidad, como algunos conocidos futbolistas, que se convierten en un auténtico referente social a seguir. Estos referentes potenciados por y desde el mercado contribuyen a reproducir jerarquías, sustentadas nuevamente en modelos ideales, por tanto inalcanzable, para la mayoría de los mortales. Lo novedoso del modelo radica en que el cuerpo masculino ahora se ha convertido también en objeto de cuidado, es decir de consumo, y esto sí que representa una novedad de un gran interés, que nos muestra la centralidad del mercado como generador y reproductor de identidades, ahora también las masculinas.

El término metrosexualidad hace referencia precisamente a la metrópoli y a los hombres metropolitanos. No es una categoría pensada precisamente para los hombres rurales, para aquellos que se alejan de los centros de decisión y de las tiendas de marca. Pero evidentemente esta referencia metropolitana no es suficiente para comprender esta nueva categoría. Un metrosexual es alguien que cuida su cuerpo y que tiene un buen cuerpo, algo para lo cual es necesaria una cierta capacidad económica. Parece claro que hoy ya es difícil construir modelos que de una u otra forma no estén vinculados con el mercado. De hecho es el mercado el que acabar por reconstruir la imagen de la familia, de los homosexuales, de los metrosexuales... Viejas fórmulas son reelaboradas estéticamente para que sean rentables económicamente. El ser se acaba vinculando con el tener y éste con la capacidad de consumo: ropa interior de Kelvin Clain, perfumes de Loewe, etc. Esta forma de entender el cuerpo entre los varones "heterosexuales", como ya hemos señalado, no se había producido hasta ahora, por lo menos hasta estos límites, pero ¿difiere mucho esta representación del ideal de hombre, salvo en cuestiones estéticas, de la que tenían los norteamericanos en los años setenta?

El modelo de metrosexualidad que se nos ha vendido como marcadamente rupturista no lo es tanto, tal y como puede verse en las numerosas guías existentes en las librerías sobre como transformarse en metrosexual (siempre que se tenga dinero, que no seas viejo, que no estés enfermo ni discapacitado, que no vivas en un pueblo, que tengas determinados niveles de formación, que no seas homosexual, mujer, etc.). Pero lo curioso es que a excepción de este modelo los nuevos varones apenas tienen otras referencias claras, de hecho la incertidumbre de los hombres ante el avance importante de las mujeres se traduce en una cierta confusión y desorientación. No es casual que en los últimos años asistamos a un importante impulso de los estudios sobre masculinidad, y también como hemos visto a la aparición de grupos de hombres que buscan espacios propios de encuentro para la reflexión y también para la acción.

\section{REFLEXIONES FINALES}

Aunque parece claro que la masculinidad es un término histórico, construido culturalmente, se nos plantea un interrogante que no es baladí: ¿hasta qué punto debemos hablar de masculinidad hegemónica y de masculinidades subalternas? Este cuestionamiento lo realizaba Vicent Marques en las Jornadas sobre Masculinidad celebradas en la Universidad Pablo de Olavide el año 2001. Efectivamente, las prácticas son distintas en función de otras variables en el seno de un colectivo determinado pero ¿podemos hablar de varios modelos de masculinidad o, dicho de otra forma, se puede hablar de distintas formas de ser un "verdadero" hombre?

Cuando se acuña la noción de masculinidades en plural supuso un avance realmente significativo, en tanto que ha contribuido de forma fundamental a desvelar los mecanismos de dominación que se han establecido entre los propios hombres y a analizar las distintas prácticas en torno a la masculinidad. Sin embargo, hablar de masculinidades, en plural, entraña varios riesgos siempre que nos refiramos a un mismo ámbito cultural. El riesgo fundamental es pensar que la masculinidad es una esencia que tiene múltiples formas, que se transforma pero que necesariamente tiene que seguir siendo. Es decir, cuando nos encontramos un colectivo que no sigue los dictados dominantes señalamos que es "otra forma de entender la masculinidad", pero la pregunta que se nos plantea es si eso es masculinidad o simplemente es otra cosa. ¿No será que estamos recurriendo a las categorías 
prefijadas para explicar "lo distinto", no será que estamos contribuyendo también a mantener una supuesta esencia por encima de las transformaciones y qué en último término seguimos justificando el orden establecido? "Si la ciencia simplifica la complejidad facilitando por ello la dominación (Valle, 1991), la sociedad selecciona los discursos científicos más proclives a reafirmar el orden establecido" (Valcuende del Río, 2002).

Por otro lado, si entendemos la masculinidad como un modelo dominante que condiciona la acción y el entendimiento, deberíamos pensar en la existencia de una única masculinidad, lo cual no es contradictorio con la idea de que los hombres son distintos o que se adapten al modelo en función de sus diferentes posibilidades. Ni tampoco es contradictorio con la idea de que el modelo de masculinidad presenta contradicciones que posibilitan su transformación, pero que idealmente podrían suponer también su propia destrucción. Sin embargo el carácter "mítico" de la masculinidad hace que se asuma como un inevitable destino de la naturaleza, al igual que "la feminidad", en función de una realidad dicotómica, que se reproduce tanto en los discursos más tradicionales como en algunos promovidos desde determinadas corrientes del pensamiento feminista.

Concebir las relaciones de género como un elemento relacional, estructural y de acción, en el que la homofobia y el miedo a "lo femenino" constituyen pilares fundamentales, nos puede permitir analizar la complejidad de acciones y reacciones que provoca. De esta forma alejamos del foco de atención los productos concretos que produce: hombres y mujeres, masculinidad y feminidad, dejando de confundir las consecuencias con las causas. Esta visión relacional sería útil para afrontar este debate, ya que nuevas formas en la relaciones de género provocarían también otros productos concretos, que probablemente escaparían a las clasificaciones que hoy entendemos como inevitables, en la línea de algunos de los planteamientos queer.

Los malos entendidos y las posibilidades de equivocar nuestro análisis se producen al poner en un mismo nivel las prácticas cotidianas y la representación ideal a través de la cual se oculta precisamente la diversidad. Pero el ocultamiento responde a un modelo único en un contexto único y en una coyuntura histórica también única. Quizás la forma de subvertir la noción de masculinidad es comenzar reconociendo, la línea propuesta por Marqués (2003) que efectivamente una buena parte de los hombres somos "menos masculinos" no es que seamos masculinos de otra forma.

\section{BIBLIOGRAFÍA}

Balandier, G., 1998. El Desorden. Ed. Gedisa, Barcelona.

Benavente, M., 2013. Intersexualidad: algunos argumentos para la deconstrucción del sistema social y jurídico dicotómico de sexos. Diversidad sexual en Iberoamérica. Coords. Valcuende del Río, J.M ${ }^{\mathrm{a}}$, M. Marco, D. Alarcón, pp. 229-238, Aconcagua: Sevilla.

Blanco, J., 2003. Las expectativas sobre los varones. Hombres. La construcción cultural de las masculinidades. Coords. Valcuende del Río, J.Mà., J. Blanco, Talasa: Madrid.

Blanco, J., 2013. ¿La masculinidad en crisis? Repensando el modelo, activando prácticas de transformación. Diversidad Sexual en Iberoamérica, pp. 255-274, Aconcagua: Sevilla.

Blanco, J., 2012. La masculinidad como factor de riesgo: una etnografía de la invisibilidad. Universidad Pablo de Olavide, Sevilla (sin publicar).

Bourdieu, P., 2000. La dominación masculina. Anagrama: Barcelona.

Cantero, P., 1996. La Quinta de Galaroza. Demófilo Nº 19: Huelva. Palabras y Rituales. Coord. Cantero, P., Fundación Machado: Sevilla.

Cantero, P., 2003. Hombrear. Modos de aprender a ser hombre. Hombres. La Construcción Cultural de las Masculinidades. Coords. Valcuende de Río, J.Ma., J. Blanco, pp. 53-66. Talasa: Madrid.

Guasch, O., 2000. La Crisis de la Heterosexualidad. Laertes: Barcelona.

Guasch, O., 2003. Ancianos, guerreros, efebos y afeminados: tipos ideales de masculinidad. En: Valcuende del Río, J.Ma ., J. Blanco, Hombres. La construcción cultural de las masculinidades. Talasa: Madrid. 
Guasch, O., 2012. Masculinidades: teorías y experiencias de discriminación. Rev. Sexología y Sociedad, 18, 4-10.

Kaufman, M., 1995. Los hombres, el feminismo y las experiencias contradictorias del poder entre los hombres. Género e Identidad. Ensayos sobre lo femenino y lo masculino. Coords. Arango, L., M. León, M. Viveros, Uniandes: Santa Fe de Bogotá.

Kimmel, M., 1997. Homofobia, temor, vergüenza y silencio en la identidad masculina. Masculinidades, poder y crisis. Coords. Valdés, T., J. Olivarría, pp. 49-62. Ed. de las Mujeres: Santiago de Chile.

Llamas, R., 1998. Teoría torcida. Prejuicios y discursos en torno a la "homosexualidad". Siglo XXI: Madrid.

MacKinnon, C., 1987. Sexuality. Toward a feminist theory of the state. Publicado por Harvard University Press, USA, pp. 127-154. Artículo traducido al castellano por el Centro de Derechos Humanos, Facultad de Derecho, Universidad de Chile. En: http://www.programamujerescdh.cl/ media/publicaciones/pdf/18/53.pdf.

Marqués, J.V., 2003. ¿Qué masculinidades? Hombres. La construcción cultural de las masculinidades. Coords. Valcuende del Río, J.Mª ., J. Blanco, Aconcagua: Sevilla, pp. 187-203.

Mead, M., 1973 Sexo y temperamento en las sociedades primitivas. Laia: Barcelona.

Misael, O., 2007. Estudios sobre masculinidades: aportes desde América Latina. Rev. Antropología Experimental, Texto 12, 153-160.

Sabuco, A., J.M. Valcuende del Río, 2003. La homosexualidad como imagen hiperbólica de la masculinidad. Hombres. La construcción cultural de las masculinidades, pp.135-155. Coords. Valcuende del Río, J.M'. ${ }^{\mathrm{a}}$, J. Blanco, Talasa: Madrid.

Val de Almeida, M., 2000. Senhores de Si. Uma interpretaçâo Antropológica da Masculinidade. Fim de Século, $2^{\mathrm{a}}$ ed: Lisboa.

Valcuende del Río, J.Ma ., 1999. Desde a devoção como um jogo ate a reproduão da norma social. Educação, Sociedade \& Culturas, 12, 85-98.

Valcuende del Río, J.Ma. , 2002. Ciencia, deseo y sexualidad en tres actos. Malaespina. Revista de la Facultad de Humanidades. Universidad Pablo de Olavide.

Valcuende del Río, J.Ma ${ }^{\text {. }, ~ 2004 . ~ C u e r p o s, ~ g e ́ n e r o s ~ y ~ s e x u a l i d a d e s: ~ r e p r e s e n t a c i o n e s ~ y ~ p r a ́ c t i c a s ~}$ sociales. $R$. Crítica Jurídica, 23, 149-174. Revista Latinoamericana de Política, Fisolosofía y Derecho.

Valcuende del Río, J.Mª., 2006. De la heterosexualidad a la ciudadanía. AIBR. Rev. Antropología Iberoamericana en Red, 1(1).

Valcuende del Río, J.Mª., 2010. Sexo entre hombres: los límites de la masculinidad. Rev. Int. Ciencias Sociales y Humanidades (SOCIOTAM), 20(1), 11-37. 